ISEP XIV

Correspondence

Jan Pawlowski

jan.pawlowski@zoo.unige.ch

\section{Foraminifera and Cercozoa share a common origin according to RNA polymerase II phylogenies}

\author{
David Longet, ${ }^{1}$ John M. Archibald, ${ }^{2}$ Patrick J. Keeling ${ }^{2}$ and Jan Pawlowski ${ }^{1}$ \\ ${ }^{1}$ Dept of zoology and animal biology, University of Geneva, Sciences III, 30 Quai Ernest \\ Ansermet, CH 1211 Genève 4, Switzerland \\ ${ }^{2}$ Canadian Institute for Advanced Research, Department of Botany, University of British \\ Columbia, \#3529-6270 University Blvd, Vancouver, British Columbia, Canada V6T 1 Z4
}

\begin{abstract}
Phylogenetic analysis of small and large subunits of rDNA genes suggested that Foraminifera originated early in the evolution of eukaryotes, preceding the origin of other rhizopodial protists. This view was recently challenged by the analysis of actin and ubiquitin protein sequences, which revealed a close relationship between Foraminifera and Cercozoa, an assemblage of various filose amoebae and amoeboflagellates that branch in the so-called crown of the SSU rDNA tree of eukaryotes. To further test this hypothesis, we sequenced a fragment of the largest subunit of the RNA polymerase II (RPB1) from five foraminiferans, two cercozoans and the testate filosean Gromia oviformis. Analysis of our data confirms a close relationship between Foraminifera and Cercozoa and points to Gromia as the closest relative of Foraminifera.
\end{abstract}

\section{INTRODUCTION}

Foraminifera are common marine protists characterized by granular and highly anastomosed pseudopodia (granuloreticulopodia) and, typically, an organic, agglutinated or calcareous test. They are present in all types of marine environments, but can also be found in freshwater (Pawlowski et al., 1999) and terrestrial (Meisterfeld et al., 2001) habitats. Granuloreticulopodia appear to be the shared character defining all Foraminifera regardless of their habitat and presence or absence of a test. Molecular phylogenetic studies that were mainly based on rDNA gene sequences confirmed the monophyly of Foraminifera, yet their relationships with other eukaryotes remain enigmatic.

Phylogenetic analysis of partial LSU rDNA sequences placed Foraminifera close to slime moulds (Dictyostelium, Physarum) and Entamoeba in the eukaryotic tree (Pawlowski et al., 1994). According to small-subunit (SSU) rDNA sequences, Foraminifera branch near the base of the eukaryotic tree, between the amitochondriate diplomonads and Euglenozoa (Pawlowski et al., 1996). Such an early origin contrasts with the relatively late appearance of Foraminifera in the fossil record (about 540 million years ago). Sogin (1997) proposed that the position of Foraminifera is biased by very rapid rates of rDNA evolution that

This paper was presented at the XIVth meeting of the International Society for Evolutionary Protistology in Vancouver, Canada, 19-24 June 2002.

Published online ahead of print on 5 September 2003 as DOI 10.1099/ijs.0.02597-0.

Abbreviations: ML, maximum likelihood; SSU, small-subunit. produces an artificial grouping of Foraminifera with early protist lineages. The long-branch attraction phenomenon was suggested to be responsible for the position of Foraminifera and some other putatively ancient groups of protists in rDNA trees (Philippe \& Adoutte, 1998).

The first protein data obtained from Foraminifera confirmed the artefactual character of their position in rDNA phylogenies. Analysis of actin-coding genes placed Foraminifera in a more derived position in the eukaryotic tree (Pawlowski et al., 1999). A more recent study of actin genes suggested that Foraminifera are closely related to Cercomonas and to the chlorarachniophytes Chlorarachnion and Lotharella (Keeling, 2001), a relationship that has also been suggested based on the presence of a shared insertion in polyubiquitin genes (Archibald et al., 2003). These genera are included in the Cercozoa, a heterogeneous assemblage which also comprises filosean testate amoebae (Testaceafilosia), some marine nanoflagellates, and plasmodiophorid plant pathogens (Bhattacharya et al., 1995; Bulman et al., 2001; Cavalier-Smith \& Chao, 1996/7; Kühn et al., 2000). Cercozoa are defined on a molecular level only, but their grouping is well supported by several genes, including rDNA, alpha- and beta-tubulin, and actin (Cavalier-Smith, 1998, 2000; Keeling et al., 1998, 1999; Keeling, 2001; Wylezich et al., 2002).

Recently, Burki et al. (2002) demonstrated a close relationship between Cercozoa and Gromia oviformis, a common marine protist characterized by a large organic test and smooth filose pseudopodia. In their SSU rDNA tree, Gromia is not related to other filosean testate amoebae and it branches early among Cercozoa. The taxonomic 
position of Gromia has always been considered problematic (Hedley, 1958). Because their tests show a gross morphological similarity to that of some allogromiid Foraminifera, the genus has for a long time been considered to be a member of Foraminifera (Arnold, 1972). On the other hand, Gromia has also been classified among Filosea, based on the presence of filose, non-anastomosing pseudopodia (Bovee, 1985). In the most recent protist classification, Gromia is placed among amoebae of uncertain affinities (Patterson et al., 2000).

To determine the relationships between Foraminifera, Cercozoa and Gromia, we sequenced a fragment of the largest subunit of the RNA polymerase II (RPB1) for eight representatives of these groups. Although the taxonomic sampling of RPB1 is still sparse, sequences are available for many of the higher-level eukaryotic taxa. RPB1 appears to be an alternative molecular marker useful for resolving some deep-level relationships between eukaryotic groups and the gene is considered to be present in a single-copy in most eukaryotic genomes (Klenk et al., 1995). RPB1 was successfully used to confirm the phylogenetic relationship of Microsporidia and fungi (Hirt et al., 1999). The sister relationship between red algae and green plants has been addressed using RPB1 (Stiller \& Hall, 1997; Stiller et al., 2001) and recently, Dacks et al. (2002) inferred a large-scale RPB1 phylogeny of eukaryotes. This report provides additional evidence for a close relationship of Foraminifera and Cercozoa.

\section{METHODS}

Cell culture, collection and DNA extraction. Three different Foraminifera, Reticulomyxa filosa, Allogromia sp. A and Allogromia sp. F were maintained in laboratory cultures, as described in Pawlowski et al. (1994, 1999). Other Foraminifera, Ammonia beccarii and Haynesina germanica, as well as Gromia oviformis originated from natural populations collected at Le Boucanet (France), Westhoek (The Netherlands) and the Arctic island of Spitzbergen, respectively. DNAs of Foraminifera and Gromia were isolated from approximately 100 adult specimens per species except for Allogromia sp. F, where the extraction was performed on a single gametogenic specimen. In the case of Reticulomyxa, pelleted cells were used for DNA extraction and all DNAs were extracted using the DNeasy Plant Mini kit (Qiagen).

A culture of Gymnochlora stellata (CCMP 2057) was kindly provided by $\mathrm{K}$. Ishida and maintained in $\mathrm{f} / 2$-Si medium at $20^{\circ} \mathrm{C}$ under a $16-\mathrm{h}$ light/8-h dark cycle. DNA was extracted from pelleted cells of Gymnochlora as above. DNA of Cercomonas sp. strain RS/22 (ATCC 50318) was generously provided by T. Cavalier-Smith and E. E. Chao.

Isolation of the RPB1 gene. We amplified and sequenced a $5^{\prime}$ fragment of the RPB1 gene encompassing the conserved regions A through $\mathrm{D}$, according to the secondary structure model of Cramer et al. (2001). The sequences of the primers Afor (5'-GAITGYCCIGGICAYTTYGG-3') and Drev (5'-TTCATYTCRTCICCRTCRAARTC- $\left.3^{\prime}\right)$, situated in the regions A and D, respectively, were kindly provided by J. W. Stiller. An additional primer Erev (5'-YTGYTTNCCNGTCCAYAA- $3^{\prime}$ ) was designed for the E region. Concerning Foraminifera and Gromia oviformis, the RBP1 fragment was amplified by a semi-nested PCR procedure with amplifications performed using primers Afor/Erev and re-amplifications using primers Afor/ Drev. Cercozoan RPB1 genes were amplified directly by using the Afor/Drev primers. All amplifications were carried out under standard profiles and all bands large enough to contain a viable RPB1 5' amplification product were isolated, cloned and sequenced in both directions.

Phylogenetic analyses. New sequences and existing homologues retrieved from public databases were aligned using CLUSTAL W (Thompson et al., 1994) and further adjusted by eye. Phylogenies were inferred from amino acid alignments using distance and protein maximum-likelihood. The RPB1 homologous sequence of an archaean, Sulfolobus solfataricus, was used as an outgroup. The total alignment used in this study consists of 37 taxa and 283 unambiguously aligned amino acid positions. Maximum-likelihood distances were calculated with TREE-PUZZLE 5.0 (Strimmer \& von Haeseler, 1996) using the WAG substitution frequency matrix, amino acid composition estimated from the data, and site-to-site rate variability modelled on a gamma distribution where the shape parameter alpha was estimated using eight rate categories plus invariable sites. Trees were inferred from these distance matrices using weighted neighbour-joining with WEIGHBOR (Bruno et al., 2000) and FitchMargoliash with FITCH (Felsenstein, 1993) using global rearrangements and 10 input order jumbles; 100 bootstrap replicates were carried out with each method using PUZZLEBOOT (shell script by A. J. Roger and M. Holder: http://www.tree-puzzle.de) with the alpha parameter and proportion of invariable sites taken from the original data. Protein maximum-likelihood trees were inferred using ProML (Felsenstein, 1993) using the JTT substitution frequency matrix, global rearrangements, and 10 input order jumbles. Site-tosite rate variation was modelled using the $-\mathrm{R}$ option and seven categories (corresponding to six rate categories plus invariable sites), with rates and frequencies estimated by TREE-PUZZLE as above.

\section{RESULTS AND DISCUSSION}

The length of the sequenced RPB1 gene fragments range from 1226 to 1271 nucleotides in Foraminifera, and is 1255 nucleotides in Gromia oviformis. The fragment is much longer in Gymnochlora stellata and Cercomonas ATCC 50318, for which 1393 and 1768 nucleotides were sequenced respectively. The size differences of RPB1 genes are the result of the presence of introns: two occur in Gymnochlora and five are present in Cercomonas ATCC 50318. No introns were found in the Foraminiferan and Gromia RPB1s. Several clones were sequenced for each species and no variability was observed. Distance trees were inferred initially using a dataset of 39 sequences that included the nucleomorph RPB1 genes from Chlorarachnion CCMP 621 and Lotharella amoeboformis (CCMP 2058) but the nucleomorph sequences were determined to be extremely divergent and offered little information (data not shown). As they were not relevant to the evolution of the host lineage, they were excluded from the analysis and a dataset of 37 sequences (shape parameter of 1.22 and proportion of invariable site of $0 \cdot 11$ ) was used for more comprehensive analysis.

Maximum-likelihood analysis of 37 RPB1 sequences reveals a clade consisting of Foraminifera, Gromia and Cercozoa (Fig. 1). This group is supported by bootstrap values of 84 and $72 \%$ in weighted neighbour-joining (WNJ) and Fitch-Margoliash (FM) distance trees, respectively. Within 


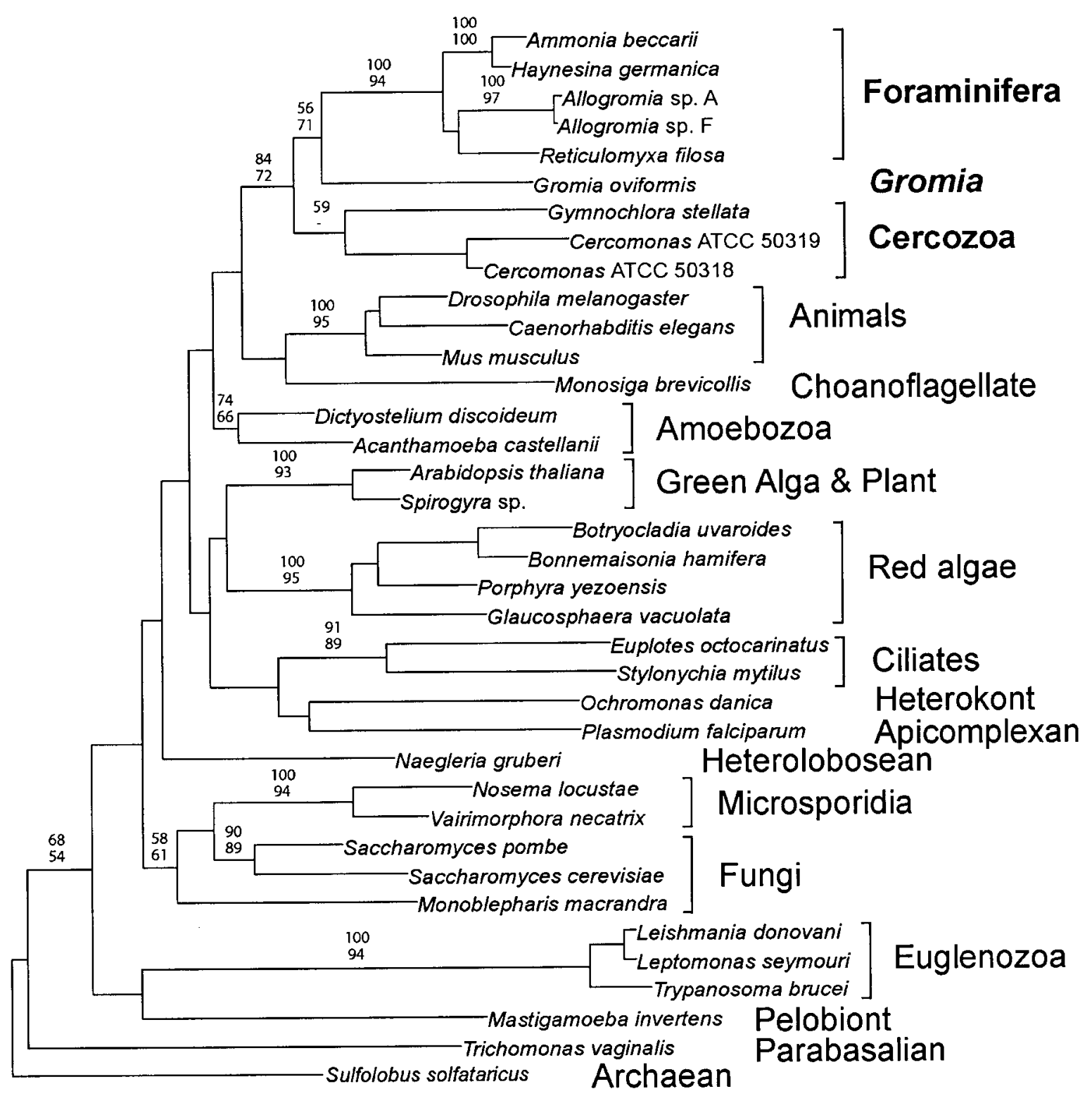

0.1

Fig. 1. Maximum-likelihood tree of eukaryotes based on partial RPB1 sequences. The tree was inferred using ProML (see text). Values at nodes indicate bootstrap support (100 replicates) greater than $50 \%$ calculated by PUZZLEBOOT on trees constructed with WEIGHBOR (top) and Fitch-Margoliash (bottom) distance methods. Except for Foraminifera, within-group bootstrap supports are omitted for clarity. A dash on the branch leading to Cercozoa indicates that the topology shown is not supported in the Fitch-Margoliash tree. The scale bar represents $0 \cdot 1$ substitution per site.

this clade, Foraminifera, Gromia and Cercozoa form three independent lineages in maximum-likelihood (ML) and WNJ trees but the FM distance tree shows Foraminifera and Gromia branching within Cercozoa (data not shown). Cercozoa appeared paraphyletic in actin phylogenies (Keeling, 2001) but this topology was not supported by bootstrap. In all analyses, Gromia appears as the closest relative to Foraminifera, although support for this relationship is not high.

Maximum-likelihood (Fig. 1) and distance analyses (data not shown) identified Trichomonas, Mastigamoeba and Euglenozoa as basal lineages, a position already observed in RPB1 phylogenies inferred using longer sequences (Stiller \& 
Hall, 2002; Dacks et al., 2002). Dacks et al. (2002) showed that the early emergence of Trichomonas and Mastigamoeba in the RPB1 phylogeny resulted from long-branch attraction artefacts. In our trees, all other taxa emerge in a poorly resolved radiation and the branching order of the different groups varies between maximum-likelihood and distance methods. However, both methods identified the same groups of eukaryotes and in no case did Foraminifera branch among the earliest eukaryotic lineages as suggested by rDNA phylogenies (Pawlowski et al., 1994, 1996).

Our analyses of RPB1 sequences support eukaryotic groups either well-recognized or observed with other molecular datasets, including ciliates, green plants, Amoebozoa (excluding Mastigamoeba), Euglenozoa, animals and red algae. The relationship of Microsporidia and fungi, supported by a body of evidence (for review see Keeling \& Fast, 2002), is consistently recovered. Alveolates (ciliates + Plasmodium) and the heterokont Ochromonas form a clade with the heterokont falling within the alveolates. This is observed in all trees, although support is weak. The alveolate clade has already been recovered in RPB1 phylogenies (Stiller et al., 2001) and Dacks et al. (2002) showed a relationship between Plasmodium and Ochromonas, suggesting a higher-level grouping of alveolates and heterokonts. This deep-level association is observed with protein phylogenies (Baldauf et al., 2000; Fast et al., 2001; Harper \& Keeling 2003). Interestingly, the red and green algae were sister groups in our ML tree (Fig. 1), in contrast to the results of previous analyses of RPB1 (Stiller \& Hall, 1997; Stiller et al., 2001). In our ML analysis, opisthokonts do not form a monophyletic group and the clade consisting of fungi and microsporidia branches somewhat deeply, although this was not the case in distance trees (data not shown). In a recent RPB1 phylogeny, an opisthokont clade was observed but its support varied greatly (Stiller \& Hall, 2002; Dacks et al., 2002).

Our data confirm the close relationship between Foraminifera and Cercozoa as suggested by actin and ubiquitin sequences analysis (Keeling, 2001). The specific association of both groups is also supported by a shared insertion of one or two amino acids at the monomer-monomer junctions of polyubiquitin proteins, a feature that is unique among eukaryotes (Archibald et al., 2003). Our study does not allow us to confidently resolve the relative branching order of Foraminifera and Cercozoa. However, our RPB1 phylogeny does allow us to conclude that among the examined species, Gromia is the closest relative of Foraminifera. The specific association of Foraminifera and Gromia in our study is congruent with revised analyses of SSU rDNA sequences (Berney \& Pawlowski, 2003). This conclusion not only confirms the traditional view of a close relationship between Gromia and Foraminifera, but also supports the hypothesis of a foraminiferal origin from a filosean ancestor.

The molecular evidence for a close relationship between Gromia and Foraminifera is of crucial importance with regard to the study of foraminiferal evolution. Comparison of genes involved in cytoskeleton formation in Gromia and Foraminifera may help to identify the genetic modifications responsible for the evolution of filopodia into granuloreticulopodia. We hypothesize that additional protein data collected from Gromia and Foraminifera might show an even closer relationship between both lineages. If this is confirmed, Gromia will serve as a reliable outgroup necessary to establish higher-level relationships among Foraminifera. Up to now, comparative taxonomic studies were mostly based on rDNA genes and severely restricted by different substitution rates at higher taxonomic levels (Pawlowski et al., 1997). According to the results of our preliminary study, the RPB1 gene seems to evolve at relatively stable rates in Foraminifera. Phylogenetic relationships within the examined foraminiferal species are congruent with the morphological distinction of organicwalled (Allogromia and Reticulomyxa) and calcareous taxa (Ammonia and Haynesina). Future studies will show whether these relationships hold true with a broader taxon sampling and if the RPB1 gene can be used as a molecular clock to examine the mode and tempo of foraminiferal evolution and to establish their molecular timescale.

\section{ACKNOWLEDGEMENTS}

The authors thank L. Zaninetti for encouraging support, J. Fahrni for technical assistance, C. Berney for help with phylogenetic analyses and M. Holzmann for comments on the manuscript. T. Cavalier-Smith, E. E. Chao and K. Ishida kindly provided cercozoan DNA extracts and cultures. J. W. Stiller kindly provided sequences of amplification primers. This study was supported by the Swiss National Science Foundation grant \# 31-59145.99 (J. P.) and by a grant from the Natural Sciences and Engineering Research Council of Canada (227301-00) to P.J.K. J.M.A. is supported by postdoctoral fellowships from the Canadian Institutes of Health Research (CIHR) and the Killam Foundation (University of British Columbia). P. J. K. is a scholar of the Canadian Institute for Advanced Research, and the Michael Smith Foundation for Health Research.

\section{REFERENCES}

Archibald, J. M., Longet, D., Pawlowski, J. \& Keeling, P. J. (2003). A novel polyubiquitin structure in Cercozoa and Foraminifera: evidence for a new eukaryotic supergroup. Mol Biol Evol 20, 62-66.

Arnold, Z. M. (1972). Observations on the biology of the protozoan Gromia oviformis Dujardin. Univ Calif Publ Zool 100.

Baldauf, S. L., Roger, A. J., Wenk-Siefert, I. \& Doolittle, W. F. (2000). A kingdom-level phylogeny of eukaryotes based on combined protein data. Science 290, 972-977.

Berney, C. \& Pawlowski, J. (2003). Revised small subunit rRNA analysis provides further evidence that Foraminifera are related to Cercozoa. J Mol Evol 57, 1-8.

Bhattacharya, D., Helmchen, T. \& Melkonian, M. (1995). Molecular evolutionary analyses of nuclear-encoded small subunit ribosomal RNA identify an independent rhizopod lineage containing the Euglyphina and the Chlorarachniophyta. J Eukaryot Microbiol 42, 65-69. 
Bovee, E. C. (1985). Class Filosea Leidy, 1879. In An Illustrated Guide to the Protozoa, pp. 228-245. Edited by J. J. Lee, S. H. Hutner $\&$ E. C. Bovee. Lawrence, KS: Society of Protozoologists.

Bruno, W. J., Socci, N. D. \& Halpern, A. L. (2000). Weighted neighbor joining: a likelihood-based approach to distance-based phylogeny reconstruction. Mol Biol Evol 17, 189-197.

Bulman, S. R., Kühn, S. F., Marshall, J. W. \& Schnepf, E. (2001). A phylogenetic analysis of the SSU rDNA from members of the Plasmodiophorida and Phagomyxida. Protist 152, 43-51.

Burki, F., Berney, C. \& Pawlowski, J. (2002). Phylogenetic position of Gromia oviformis Dujardin inferred from nuclear-encoded small subunit ribosomal DNA. Protist 153, 251-260.

Cavalier-Smith, T. (1998). A revised six-kingdom of life. Biol Rev Camb Philos Soc 73, 203-266.

Cavalier-Smith, T. (2000). Flagellate megaevolution: the basis for Eukaryote diversification. In The Flagellates: Unity, Diversity and Evolution, pp 361-390. Edited by B. S. C. Leadbeater \& J. C. Green. London: Taylor \& Francis.

Cavalier-Smith, T. \& Chao, E. E. (1996/7). Sarcomonad ribosomal RNA sequences, rhizopod phylogeny, and the origin of euglyphid Amoebae. Arch Protistenkd 147, 227-236.

Cramer, P., Bushnell, D. A. \& Kornberg, R. D. (2001). Structural basis of transcription: RNA polymerase II at 2.8 angstrom resolution. Science 292, 1863-1876.

Dacks, J. B., Marinets, A., Ford Doolittle, W., Cavalier-Smith, T. \& Logsdon, J. M., Jr (2002). Analyses of RNA polymerase II genes from free-living protists: phylogeny, long branch attraction, and the eukaryotic big bang. Mol Biol Evol 19, 830-840.

Fast, N. M., Kissinger, J. C., Roos, D. S. \& Keeling, P. J. (2001). Nuclear-encoded, plastid-targeted genes suggest a common origin for apicomplexa and dinoflagellates plastids. Mol Biol Evol 18, 418-426.

Felsenstein, J. (1993). PHYLIP (Phylogeny Inference Package). Department of Genetics, University of Washington, Seattle, USA.

Harper, J. T. \& Keeling, P. J. (2003). Nucleus-encoded, plastidtargeted glyceraldehyde-3-phosphate dehydrogenase (GAPDH) indicates a single origin for chromalveolate plastids. Mol Biol Evol MBE Advance Access, http://dx.doi.org/10.1093/molbev/msg195

Hedley, R. H. (1958). Confusion between Gromia oviformis and Allogromia ovoidea. Nature 182, 1391-1392.

Hirt, R. P., Logsdon, J. M., Jr, Healy, B., Dorey, M. W., Doolittle, W. F. \& Embley, T. M. (1999). Microsporidia are related to Fungi: evidence from the largest subunit of RNA polymerase II and other proteins. Proc Natl Acad Sci U S A 96, 580-585.

Keeling, P. J. (2001). Foraminifera and Cercozoa are related in actin phylogeny: two orphans find a home? Mol Biol Evol 18, 1551-1557.

Keeling, P. J. \& Fast, N. M. (2002). Microsporidia: biology and evolution of highly reduced intracellular parasites. Annu Rev Microbiol 56, 93-116.

Keeling, P. J., Deane, J. A. \& McFadden, G. I. (1998). The phylogenetic position of alpha- and beta-tubulins from the Chlorarachnion host and Cercomonas (Cercozoa). J Eukaryot Microbiol 45, 561-570.

Keeling, P. J., Deane, J. A., Hink-Schauer, C., Douglas, S. E., Maier, U.-G. \& McFadden, G. I. (1999). The secondary endosymbiont of the cryptomonad Guillardia theta contains alpha-, beta-, and gammatubulin genes. Mol Biol Evol 16, 1308-1313.

Klenk, H.-P., Zillig, W., Lanzendörfer, M., Grampp, B. \& Palm, P. (1995). Location of protist lineages in a phylogenetic tree inferred from sequences of DNA-dependent RNA polymerases. Arch Protistenkd 145, 221-230.

Kühn, S., Lange, M. \& Medlin, L. K. (2000). Phylogenetic position of Cryothecomonas inferred from nuclear-encoded small subunit ribosomal RNA. Protist 151, 337-345.

Meisterfeld, R., Holzmann, M. \& Pawlowski, J. (2001). Morphological and molecular characterization of a new terrestrial allogromiid species: Edaphoallogromia australica gen. et spec. nov. (Foraminifera) from Northern Queensland (Australia). Protist 152, 185-192.

Patterson, D. J., Simpson, A. G. B. \& Rogerson, A. (2000). Amoebae of uncertain affinities. In An Illustrated Guide to the Protozoa, 2nd edn, pp. 804-827. Edited by J. J. Lee, G. F. Leedale \& P. Bradbury. Lawrence, KS: Society of Protozoologists.

Pawlowski, J., Bolivar, I., Guiard-Maffia, J. \& Gouy, M. (1994). Phylogenetic position of the Foraminifera inferred from LSU rRNA gene sequences. Mol Biol Evol 11, 929-938.

Pawlowski, J., Bolivar, I., Fahrni, J. F., Cavalier-Smith, T. \& Gouy, M. (1996). Early origin of Foraminifera suggested by SSU rRNA gene sequences. Mol Biol Evol 13, 445-450.

Pawlowski, J., Bolivar, I., Fahrni, J. F., de Vargas, C., Gouy, M. \& Zaninetti, L. (1997). Extreme differences in rates of molecular evolution of foraminifera revealed by comparison of ribosomal DNA sequences and the fossil record. Mol Biol Evol 14, 498-505.

Pawlowski, J., Bolivar, I., Fahrni, J. F., de Vargas, C. \& Bowser, S. S. (1999). Molecular evidence that Reticulomyxa filosa is a freshwater naked foraminifer. J Eukaryot Microbiol 46, 612-617.

Philippe, H. \& Adoutte, A. (1998). The molecular phylogeny of eukaryotes: solid facts and uncertainties. In Evolutionary Relationships Among Protozoa, pp 25-56. Edited by H. Coombs, K. Vickerman, M. A. Sleigh \& A. Warren. London: Chapman \& Hall.

Sogin, M. (1997). History assignment: when was the mitochondrion founded? Curr Opin Genet Dev 7, 792-799.

Stiller, J. W. \& Hall, B. D. (1997). The origin of red algae: implications for plastid evolution. Proc Natl Acad Sci U S A 94, 4520-4525.

Stiller, J. W. \& Hall, B. D. (2002). Evolution of the RNA polymerase II C-terminal domain. Proc Natl Acad Sci U S A 99, 6091-6096.

Stiller, J. W., Riley, J. \& Hall, B. D. (2001). Are red algae plants? A critical evaluation of three key molecular data sets. J Mol Evol 52, 527-539.

Strimmer, K. \& von Haeseler, A. (1996). Quartet puzzling: a quartet maximum-likelihood method for reconstructing tree topologies. Mol Biol Evol 13, 964-969.

Thompson, J. D., Higgins, D. G. \& Gibson, T. J. (1994). CLUSTAL W: improving the sensitivity of progressive multiple sequence alignment through sequence weighting, position-specific gap penalties and weight matrix choice. Nucleic Acids Res 22, 4673-4680.

Wylezich, C., Meisterfeld, R., Meisterfeld, S. \& Schlegel, M. (2002). Phylogenetic analyses of small subunit ribosomal RNA coding regions reveal a monophyletic lineage of euglyphid testate amoebae (Order Euglyphida). J Eukaryot Microbiol 49, 108-118. 